\title{
NEFL Pro22Arg mutation in Charcot-Marie-Tooth disease type 1
}

\author{
Ji Soo Shin · Ki Wha Chung · Sun Young Cho • \\ Jiyoung Yun · Su Jin Hwang · Sung Hee Kang • \\ En Min Cho $\cdot$ Seung-Min Kim $\cdot$ Byung-Ok Choi
}

Received: 13 May 2008/ Accepted: 24 June 2008/Published online: 29 August 2008

(C) The Japan Society of Human Genetics and Springer 2008

\begin{abstract}
Charcot-Marie-Tooth disease (CMT) is classified into demyelinating neuropathy (CMT1) and axonal neuropathy (CMT2). Mutations in the neurofilament light chain polypeptide $(N E F L)$ gene are present in CMT2E and CMT1F neuropathies. Two types of Pro22 mutations have been previously reported: Pro22Ser in CMT2E with giant axons, and Pro22Thr in CMT1F. In this study, we identified another Pro22 mutation, Pro22Arg, in a Korean CMT1 family. An investigation to identify the clinical and pathological characteristics of the Pro22Arg revealed that it is associated with demyelinating neuropathy features in CMT1F. Histopathological findings showed onion bulb formations but no giant axons. It appears that the Pro22 mutations may influence not only the Thr-Pro phosphorylation site by proline-directed protein kinases but also other structural alteration of the NEFL protein in a different way.
\end{abstract}

Keywords Peripheral neuropathy .

Charcot-Marie-Tooth disease type 1F (CMT1F) .

Neurofilament light chain polypeptide (NEFL) .

Pro22Arg

J.S. Shin and K.W. Chung contributed equally to this work.

J. S. Shin · S. Y. Cho · J. Yun · S. J. Hwang - B.-O. Choi ( $ه)$

Department of Neurology and Ewha Medical Research Center,

Ewha Womans University, School of Medicine,

Mokdong Hospital, 911-1 Mokdong Yangcheon-ku,

Seoul 158-710, South Korea

e-mail: bochoi@ewha.ac.kr

K. W. Chung $\cdot$ S. H. Kang $\cdot$ E. M. Cho

Department of Biological Science,

Kongju National University, Gongju, South Korea

S.-M. Kim

Department of Neurology, Yonsei University,

College of Medicine, Seoul, South Korea

\begin{abstract}
Abbreviations
CMT Charcot-Marie-Tooth disease

NCV Nerve conduction velocity

NEFL Neurofilament light chain

polypeptide

PDPK Proline-directed protein kinase
\end{abstract}

\section{Introduction}

Charcot-Marie-Tooth disease (CMT) is the most common inherited motor and sensory neuropathy and is divided into demyelinating (CMT1) and axonal (CMT2) forms using electrophysiological and pathological criteria. CMT1 is characterized by demyelination and slow nerve conduction velocities (NCVs), whereas CMT2 is characterized by signs of axonal regeneration and normal or slightly reduced NCVs (Harding and Thomas 1980).

Neurofilament light chain polypeptide (NEFL), which consists of an N-terminal head, a central road, and a C-terminal tail domain, is one of the most abundant cytoskeletal components in the neuron (Brownlees et al. 2002). Several missense mutations in the $N E F L$ gene have been reported, and a number of these have been predicted to produce alterations in the formation of the intermediate filament network in neurons (Pérez-Ollé et al. 2005; Sasaki et al. 2006). Mutations in the NEFL gene were originally reported to be associated with CMT2E (De Jonghe et al. 2001; Fabrizi et al. 2004). However, because some patients had markedly reduced NCVs, it was suggested that $N E F L$ neuropathy was also relevant with CMT1F (Jordanova et al. 2003).

Pathologic characterizations have been performed in only a small number of NEFL mutations. Three mutations (Pro22Ser, Leu268Pro and del322Cys-326Asn) have been 
reported to be associated with giant axonal neuropathies and secondary demyelination (Fabrizi et al. 2004, 2007), whereas the other mutations (Glu89Lys and Glu397Lys) were found to be associated with onion bulb formations and loss of large myelinated fibers, but not giant axonal neuropathy (Jordanova et al. 2003; Züchner et al. 2004).

Particularly, two Pro22 mutations have been reported: Pro22Ser was found in CMT2E with giant axons in the sural nerve (Fabrizi et al. 2004; Georgiou et al. 2002), and Pro22Thr was found to be associated with CMT1F (Yoshihara et al. 2002). It has been suggested that Pro22 mutations abolish the Thr-Pro phosphorylation sequence of the head domain of NEFL by proline-directed protein kinases (PDPKs) (Sasaki et al. 2006). In this study, we identified another NEFL Pro22Arg mutation, which was not reported in the inherited peripheral neuropathies mutation database (http://www.molgen.ua.ac.be/CMTMutations), and sought to determine its clinical and pathological characteristics.

\section{Patients and methods}

\section{Clinical presentations}

Patient 1: The proband (F/41 years; Fig. 1a, II-4) of the FC99 family complained of a gait difficulty and lower limb weakness. She first felt to have a gait difficulty at 13 years of age (Table 1). She frequently fell, and showed progressive impairment. At 19 years of age, wasting and weakness of bilateral hand muscles were evident, and at 34 years, she wore a foot brace. She did not display any signs of hoarseness or cranial nerve involvement. A neurological examination at 41 years showed distal wasting and muscle weakness in the lower limbs and both hands, with proximal thigh muscle involvement. Vibration and pain perceptions were reduced in the distal upper and lower limbs, and all muscle stretch reflexes were absent. At 34 years, her median motor nerve conduction velocity (MNCV) was $26.1 \mathrm{~m} / \mathrm{s}$ and compound muscle action potential (CMAP) $0.1 \mathrm{mV}$, and ulnar MNCV was $25.3 \mathrm{~m} / \mathrm{s}$ CMAP $4.7 \mathrm{mV}$. However, CMAPs of peroneal and posterior tibial nerves were not detectable. Sensory nerve action potentials (SNAPs) of median, ulnar, and sural nerves were absent. Moreover, at 41 years, motor and SNAPs including median, ulnar, peroneal, posterior tibial, and sural nerves were undetectable. Brainstem auditory evoked potentials and visual evoked potentials were normal. Her father (I-1) who died at 83 years, had complained of walking difficulty and become wheel-chair bound at 62 years, but her mother (F/73 years; I-2) remains healthy. Her elder sister (F/51 years; II-1) and younger brother (M/37 years; II-6) were able to walk with foot braces.

Patient 2: The son of patient 1 (M/16 years; Fig. 1a, III-3) was admitted due to weakness of distal lower limbs. He began to experience progressive gait abnormalities at 3 years of age and experienced symptoms of hand muscle atrophy at (a)

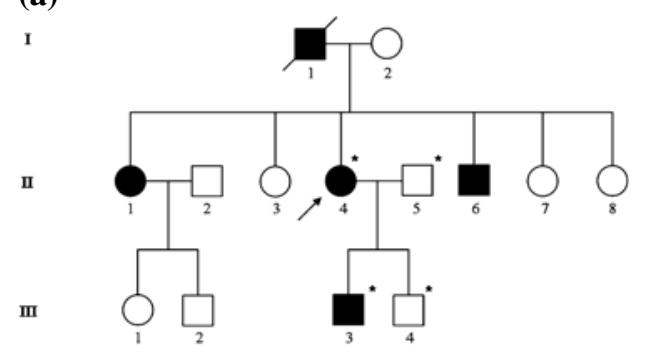

(b)

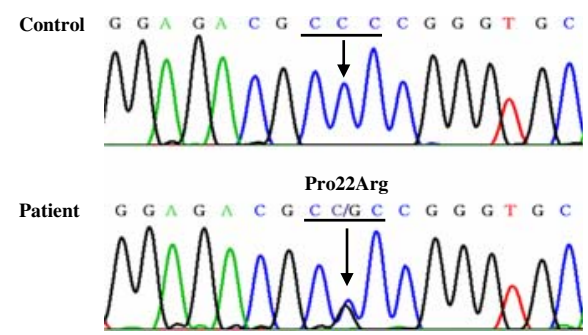

(c)

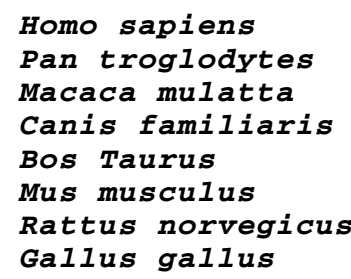

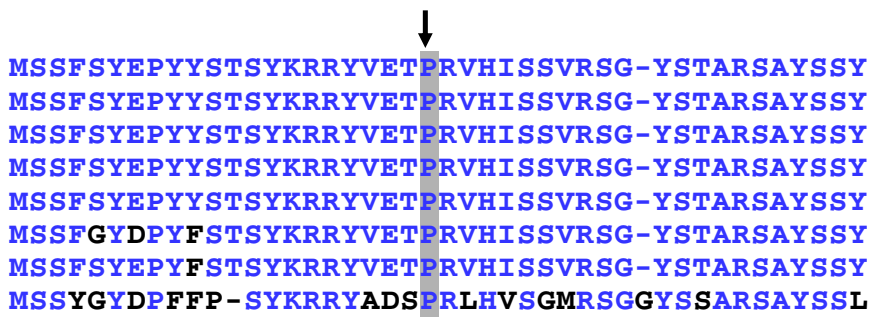

Fig. 1 Pedigree and mutational analysis of the FC99 family with NEFL Pro22Arg mutation. a The open symbols represent unaffected males (open square) and unaffected females (open circle), and the filled symbols affected males (filled square) and affected females (filled circle). The arrow indicates the proband, and asterisks indicate the available deoxyribonucleic acid (DNA) samples. b Sequencing chromograms of the NEFL c.65C $>\mathrm{G}$ mutation. The 22nd codon CCC encoding Pro in the normal sample was replaced by CGC encoding Arg in the patient sample. c Conservation of Pro22 in different species. Multiple alignments of amino acid sequences demonstrate that the mutation site is well conserved between different species 
Table 1 Clinical and electrophysiological features of Charcot-Marie-Tooth patients with NEFL Pro22 mutations

\begin{tabular}{|c|c|c|c|c|}
\hline Mutations & Pro22Arg & Pro22Thr & Pro22Ser & \\
\hline CMT phenotype & CMT1 & CMT1 & СMT2 & CMT2 \\
\hline Inheritance & $\mathrm{AD}$ & $\mathrm{AD}$ & $\mathrm{AD}$ & $\mathrm{AD}$ \\
\hline Age of onset (years) & $3-13$ & $18-24$ & $<10$ & $<10$ to 38 \\
\hline Muscle weakness $^{\mathrm{a}}$ & ++ to ++++ & ++ to +++ & + to ++ & + to ++ \\
\hline Muscle atrophy & $\mathrm{U}<\mathrm{L}$ & $\mathrm{U}<\mathrm{L}$ & $\mathrm{U}<\mathrm{L}$ & $\mathrm{U}<\mathrm{L}$ \\
\hline Sensory loss & Yes & Yes & Yes & Yes \\
\hline Reflexes & A & ND & $\mathrm{D}, \mathrm{A}$ & A \\
\hline Pes cavus & Yes & ND & Yes & Yes \\
\hline Median MNCV (m/s) & $22-29$ & $29-36$ & $21-54$ & $21-43$ \\
\hline Median CMAP (mV) & $0.1-3.7$ & $0.01-0.74$ & $0.8-4.6$ & $2.0-7.4$ \\
\hline Median SNCV (m/s) & $21-27$ & 24 & NP & NP \\
\hline Median SNAP $(\mu \mathrm{V})$ & $4.2-5.3$ & 0.7 & NP & NP \\
\hline Pathologic findings & Onion bulbs & ND & ND & Giant axons \\
\hline References & This study & Yoshihara et al. (2002) & Georgiou et al. (2002) & Fabrizi et al. (2004) \\
\hline
\end{tabular}

Normal values: median $\mathrm{MNCV} \geq 51 \mathrm{~m} / \mathrm{s}, \mathrm{SNCV} \geq 40 \mathrm{~m} / \mathrm{s}, \mathrm{CMAP} \geq 6.0 \mathrm{mV}$, and $\mathrm{SNAP} \geq 8.8 \mu \mathrm{V}$

$A D$ autosomal dominant, $U$ upper limb, $L$ lower limb, $D$ diminished, $A$ absent, $N D$ no data, $N P$ no potential, $M N C V$ motor nerve conduction velocity, $S N C V$ sensory nerve conduction velocity, $C M A P$ compound muscle action potential, SNAP sensory nerve action potential

${ }^{a}$ Weakness, + ankle dorsiflexion more than grade 4 Medical Research Council (MRC), ++ ankle dorsiflexion less than grade 4 MRC, +++ ankle dorsiflexion less than grade $4 \mathrm{MRC}$ and proximal weakness, ++++ ankle dorsiflexion less than grade 4 MRC and wheelchair bound

10 years of age (Table 1). He had a steppage gait with foot dorsiflexion weakness, pes cavus, and hammer toes but was able to walk without assistance. A neurological examination at 16 years revealed moderate weakness and atrophy of the distal muscles of upper and lower limbs. All sensory modalities were impaired distally in the upper and lower limbs, and stretch reflexes were absent. In the 3 years between 13 and 16 years of age, his median MNCVs ranged from 21.6 to $28.6 \mathrm{~m} / \mathrm{s}$ and CMAPs from 1.5 to $3.7 \mathrm{mV}$, and ulnar MNCVs ranged from 23.6 to $24.6 \mathrm{~m} / \mathrm{s}$ and CMAPs from 1.3 to $6.9 \mathrm{mV}$. In addition, median sensory NCVs ranged from 21.0 to $27.1 \mathrm{~m} / \mathrm{s}$ and SNAPs from 4.2 to $5.3 \mu \mathrm{V}$. However, sural nerve SNAPs were not detectable.

\section{Mutational analysis}

Genomic DNA was extracted from whole blood samples from a Korean FC99 family using QIAamp deoxyribonucleic acid (DNA) blood kits (Qiagen, Germany). A group of 210 healthy controls were also recruited. All participants provided informed consent. The mutation was screened by DNA sequencing of all exons and contiguous flanking intronic sequences. The primer sequences and polymerase chain reaction (PCR) conditions of 17p11.2-p12 duplication/deletion, NEFL, Cx32, EGR2, MPZ, and PMP22 were followed by Choi et al. (2004), and PCR conditions of DNM2, MFN2, HSP27, or HSP22 genes are available on request. PCR products were purified using EXOSAP-IT kits (USB, USA), and nucleotide sequences were determined using an automatic genetic analyzer ABI3100 using a big dye terminator cycle sequencing ready reaction kit (Applied Biosystems, USA). We used the SeqScape (Ver. 2.1) program (Applied Biosystems) to detect the sequence variation. We confirmed the sequence variations by analyzing both DNA strands.

\section{Pathologic studies}

The proband (II-4) underwent sural nerve biopsy at 41 years. Pathological examinations included light and electron microscopic analysis and teasing. One sural nerve fragment was fixed in 10\% formalin, embedded in paraffin, and stained with hematoxylin-eosin. Another fragment was immediately fixed by immersion in 5\% buffered glutaraldehyde and postfixed in $1 \%$ osmium tetroxide. Eponembedded semithin and ultrathin sections were prepared for light and ultrastructural examinations. About 100 single myelinated fibers were teased from the nerve biopsy sample.

\section{Results}

Molecular genetic analysis

We identified a missense mutation c.65C $>\mathrm{G}$, which resulted in Pro22Arg in the FC99 family with CMT1 phenotype (Fig. 1b). This mutation was found in the 
proband (II-4) and her oldest son (III-3) but was not found in her husband (II-5) or youngest son (III-4). This mutation was well cosegregated with affected members in the autosomal dominant pattern and was not present in the 210 healthy controls. The Pro22Arg missense mutation is located in the N-terminal head domain of the NEFL protein. Mutations have been previously reported in the same 22nd codon: c.64C $>$ A resulting in Pro22Thr (Yoshihara et al. 2002) and c.64C $>\mathrm{T}$ resulting in Pro22Ser (Fabrizi et al. 2004; Georgiou et al. 2002), but this Pro22Arg mutation was not reported in the inherited peripheral neuropathies mutation database. Amino acids at the mutated site are highly conserved in different species (Fig. 1c). No 17p11.2-p12 duplication/deletion or causative mutations in the $C x 32$ (GJB1), EGR2, MPZ (PO), DNM2, PMP22, MFN2, HSP27, or HSP22 genes were detected in the FC99 family.

\section{Neuropathology}

A histogram demonstrated a marked reduction of largediameter fibers in the proband. The diameter of the largest myelinated fiber was $5.8 \mu \mathrm{m}$, and the fiber density was 9,570 per $\mathrm{mm}^{2}$ (range in controls $=6,500-9,500$ per $\mathrm{mm}^{2}$ ). In semithin sections, onion bulbs were recognized, and numerous small irregular foldings of myelin sheaths were observed (Fig. 2a). However, no giant axons or axonal swellings were found. Electron microscopy confirmed the loss of large myelinated fibers and revealed that onion bulbs were composed of circular or nearly closed layers of extended Schwann cell processes (Fig. 2b, c). No focal swellings were observed in teased fibers.

\section{Discussion}

Mutations in the NEFL gene result in CMT neuropathies with variable clinical and pathological expressions. In this study, we identified a Pro22Arg (c.65C > G) mutation in a CMT1 family. We believed that this mutation was the underlying cause of the CMT1 phenotype in the FC99 family for the following reasons: cosegregation of the mutation with affected members of the pedigree, no similar mutation in controls $(n=210)$, well-conserved Pro22 among different species, and the findings of previous reports on the effect of different mutations at the Pro22 site. It seems that codon 22 is one of the mutational hot spots in the NEFL gene because three different Pro22 mutations, including the one found in this study, have been reported (Fabrizi et al. 2004; Georgiou et al. 2002; Yoshihara et al. 2002).

Three different families have been reported to have Pro22 mutations: Pro22Ser in a Slovenian family (Georgiou et al. 2002) and an Italian family (Fabrizi et al. 2004), and Pro22Thr in a Japanese family (Yoshihara et al. 2002). Histopathological features were only described in a Pro22Ser patient in an Italian family and featured giant axons with neurofilament accumulation (Fabrizi et al. 2004). However, the Japanese family with the Pro22Thr mutation was reported to have the CMT1 phenotype based on clinical and electrophysiological features (Yoshihara et al. 2002). Moreover, the FC99 family with Pro22Arg mutation also showed a CMT1 neuropathy with onion bulb formations in this study. Thus, phenotypic variations for Pro22 mutations encompass not only clinical features but also histopathologic findings.

In this study, we compared phenotypes of the Pro22Arg mutation with those of reported Pro22Ser and Pro22Thr patients (Table 1). Muscle weakness and NCV slowing were more pronounced in Pro22Thr and Pro22Arg patients than in Pro22Ser patients. Furthermore, giant axonal neuropathies were observed only in a CMT2E patient with Pro22Ser mutation. However, the proband with the Pro22Arg mutation did not show giant axons but did show onion bulbs. Although further pathologic studies are needed, the presence of giant axons and onion bulbs could be useful for distinguishing demyelinating and axonal neuropathy due to NEFL mutations.

In previous studies, Pro22Ser and Pro22Thr mutants showed defects in filamentous network formation irregular aggregation in the SW13- cell, and the mutations abolish the
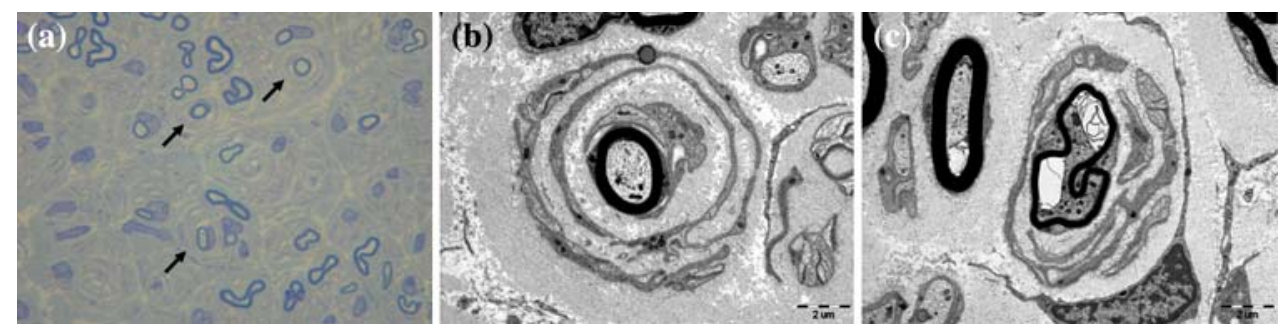

Fig. 2 Neuropathological examination of a sural nerve biopsy performed in patient 1 (II-4). a Light microscopy of the sural nerve. Transverse semithin section revealed several thinly myelinated fibers and onion bulb formations (arrows), but giant axons and axonal swellings were not observed. A marked reduction in the number of large-diameter fibers was evident (toluidine blue, original magnification $\times 400$ ). b, c Electron microscopy. The photograph shows an onion bulb formation surrounded by circular layers of multiple flat Schwann cell processes (b) and small irregular foldings of myelin sheaths surrounded by multiple layers (c) 
Thr-Pro PDPK phosphorylation site in the NEFL head domain, which regulates filament assembly via phosphorylation (Pérez-Ollé et al. 2005; Sasaki et al. 2006). In our study, patients with the Pro22Arg mutation had the same demyelinating neuropathies as did patients with the Pro22Thr mutation; therefore, it seems that the Pro22Arg mutation also may abolish the Thr-Pro phosphorylation site of the head domain by PDPKs. However, because the Pro22Ser mutation revealed a different phenotype from the Pro22Thr and Pro22Arg mutations, it is likely that the Pro22 mutations may influence not only on the phosphorylation site but also other structural alterations of the NEFL protein in a different way.

Mutations of Pro to Ser, Thr or Arg at the same codon 22 site in the NEFL gene can result in different clinical presentations of CMT1 or CMT2. Different phenotypic variations have also been observed in the mutations at codon 8 in the head domain or at codon 397 in the coil $2 b$ domain of the NEFL protein. Thus, it is interesting that in $N E F L$ mutations, specific amino acid changes even in the same codon result in different CMT phenotypes.

Acknowledgments This work was supported by the Brain Korea 21 project, the Korea Research Foundation Grant funded by the Korean Government (MEST) (KRF-2007-313-E00397), and the Star Project at Kongju National University.

\section{References}

Brownlees J, Ackerley S, Grierson AJ, Jacobsen NJ, Shea K, Anderton BH, Leigh PN, Shaw CE, Miller CC (2002) CharcotMarie-Tooth disease neurofilament mutations disrupt neurofilament assembly and axonal transport. Hum Mol Genet 11:28372844

Choi BO, Lee MS, Shin SH, Hwang JH, Choi KG, Kim WK, Sunwoo IN, Kim NK, Chung KW (2004) Mutational analysis of PMP22, $M P Z, G J B 1, E G R 2$ and NEFL in Korean Charcot-Marie-Tooth neuropathy patients. Hum Mutat 24:185-186
De Jonghe P, Mersivanova I, Nelis E, Del Favero J, Martin JJ, Van Broeckhoven C, Evgrafov O, Timmerman V (2001) Further evidence that neurofilament light chain gene mutations can cause Charcot-Marie-Tooth disease type 2E. Ann Neurol 49:245-249

Fabrizi GM, Cavallaro T, Angiari C, Bertolasi L, Cabrini I, Ferrarini M, Rizzuto N (2004) Giant axon and neurofilament accumulation in Charcot-Marie-Tooth disease type 2E. Neurology 62: 1429-1431

Fabrizi GM, Cavallaro T, Angiari C, Cabrini I, Taioli F, Malerba G, Bertolasi L, Rizzuto N (2007) Charcot-Marie-Tooth disease type 2E, a disorder of the cytoskeleton. Brain 130:394-403

Georgiou DM, Zidar J, Korosec M, Middleton LT, Kyriakides T, Christodoulou K (2002) A novel $N F-L$ mutation Pro22Ser is associated with CMT2 in a large Slovenian family. Neurogenetics 4:93-96

Harding AE, Thomas PK (1980) The clinical features of hereditary motor and sensory neuropathy types I and II. Brain 103:259-280

Jordanova A, De Jonghe P, Boerkoel CF, Takashima H, De Vriendt E, Ceuterick C, Martin JJ, Butler IJ, Mancias P, Papasozomenos SCh, Terespolsky D, Potocki L, Brown CW, Shy M, Rita DA, Tournev I, Kremensky I, Lupski JR, Timmerman V (2003) Mutations in the neurofilament light chain gene (NEFL) cause early onset severe Charcot-Marie-Tooth disease type 1A. Brain 126:590-597

Pérez-Ollé R, López-Toledano MA, Goryunov D, Cabrera-Poch N, Stefanis L, Brown K, Liem RK (2005) Mutations in the neurofilament light gene linked to Charcot-Marie-Tooth disease cause defects in transport. J Neurochem 93:861-874

Sasaki T, Gotow T, Shiozaki M, Sakaue F, Saito T, Julien JP, Uchiyama Y, Hisanaga S (2006) Aggregate formation and phosphorylation of neurofilament-L Pro22 Charcot-Marie-Tooth disease mutants. Hum Mol Genet 15:943-952

Yoshihara T, Yamamoto M, Hattori N, Misu K, Mori K, Koike H, Sobue G (2002) Identification of novel sequence variant in the neurofilament light gene in a Japanese population: analysis of Charcot-Marie-Tooth disease patients and normal individuals. J Peripher Nerv Syst 7:221-224

Züchner S, Vorgerd M, Sindern E, Schröder JM (2004) The novel neurofilament light (NEFL) mutation Glu397Lys is associated with a clinically and morphologically heterogeneous type of CharcotMarie-Tooth neuropathy. Neuromuscul Disord 14:147-157 